\title{
DEPORTE Y POLÍTICA PÚBLICA EN EL MUNICIPIO DE AVELLANEDA: ARTICULACIONES ENTRE LO LOCAL Y LO NACIONAL
}

\author{
SPORT AND PUBLIC POLICY IN THE MUNICIPALITY OF AVELLANEDA: RELATIONS BETWEEN \\ NATIONAL AND LOCAL
}

Verónica Moreira ${ }^{1}$

\section{Resumen}

Este trabajo trata sobre las políticas públicas producidas en torno al deporte en el municipio de Avellaneda, uno de los 24 distritos que rodea a la Ciudad Autónoma de Buenos Aires. El trabajo se centra en la presentación de dos programas que el Gobierno comunal ha impulsado para desarrollar el deporte entre niños, niñas y jóvenes del municipio. Los programas están destinados a la promoción del deporte en dos modalidades, que las autoridades presentan como dimensiones complementarias: el deporte recreativo y el deporte de alto rendimiento. La presentación, además, permite exponer algunas de las condiciones de cómo se practica deporte en este partido bonaerense. Finalmente, el trabajo permite observar cómo los ideales que sustentan las políticas públicas comunales se articulan con los preceptos que atraviesan las políticas del deporte del gobierno nacional.

Palabras claves: políticas públicas, deporte, Gobierno municipal, Gobierno nacional.

\section{Abstract}

This paper deals with public policies produced around sports in the municipality of Avellaneda, one of the 24 districts sorrounding the city of Buenos Aires. The paper focuses on the presentation of two programs that the city government has pushed to develop sport among children and youth from the municipality. The programs are aimed at promoting the sport in two modes: recreational sport and competitive sport. This presentation also allows exposure to some of the conditions of how this sport is practised in the Buenos Aires district. Finally, this paper allows us to observe how the communal ideals underlying public policies articulate with the precepts that cross political sport national government.

Keywords: public policy, sport, local government, national government.

Fecha de recepción: 16 de octubre de 2014

Fecha de aprobación: 23 de marzo de 2015

Para citar este artículo:

Moreira, V. (2015). Deporte y política pública en el municipio de Avellaneda: articulaciones entre lo local y lo nacional. Lúdica Pedagógica, (21), 53-61.

\footnotetext{
1 Doctora en Ciencias Sociales (UBA), magistra en Antropología Social (UNSAM) y Licenciada en Antropología (UBA). Instituto de Investigaciones Gino Germani (Facultad de Ciencias Sociales, UBA) y Consejo Nacional de Investigaciones Científicas y Técnicas. Correos electrónicos: mvemoreira@
} yahoo.com.ar, vmoreira@sociales.uba.ar 


\section{INTRODUCCIÓN}

Cuando retorné mi trabajo de campo durante 2013 para continuar con el estudio sobre las relaciones entre política tradicional y fútbol en Avellaneda -un partido del Conurbano Bonaerense, ubicado al sur del centro administrativo de la República Argentina-, me topé con un tema que no había considerado con antelación: las políticas públicas municipales destinadas al desarrollo del deporte. Sí sabía que dicho municipio contaba con la presencia de tres clubes de fútbol de la primera división y dos de las categorías del ascenso de la Asociación del Fútbol Argentino (AFA); y que esta particularidad se afianzaba con un hecho que es único en el mundo: la convivencia de dos estadios construidos a doscientos metros de distancia entre sí que, además, pertenecen a dos clubes considerados dentro de los cinco grandes del fútbol nacional, el Club Atlético Independiente y Racing Club. Las autoridades locales habían especulado, en otros tiempos, en construir en esta zona un circuito turístico con un estadio único como epicentro. Este proyecto nunca se concretó porque entre sus autores seguramente primó la mesura económica y la sensatez futbolística. En Argentina, a diferencia de otros países, hay una ecuación que tiene peso de ley entre los hinchas: un club de fútbol = un estadio.

No obstante, en materia deportiva, otros proyectos tomaron forma y se fueron cristalizando en políticas públicas definidas, en especial desde la asunción del intendente del municipio en el año 2011. A partir de dicho periodo, cobraron protagonismo dos programas que tuvieron como meta impulsar de manera complementaria el desarrollo del deporte entre los habitantes del partido. En efecto, este trabajo tiene como objetivo reflexionar sobre las políticas públicas deportivas en Avellaneda durante la última gestión de gobierno (2011-2015). Como las propuestas del municipio se sostienen sobre una serie de ideales que también caracterizan a un sector de las políticas públicas nacionales, creo conveniente presentar ciertas líneas que permiten observar la articulación entre ambas esferas: lo local y lo nacional. Por último, cabe aclarar, que los datos que sustentan el argumento de esta reflexión responden al trabajo de campo realizado entre los funcionarios del Gobierno comunal, que comenzó en 2013 y que continúa en la actualidad.

\section{Programas del gobierno municipal: Aliento y Alentar $^{2}$}

Avellaneda se ubica al sudeste de la Ciudad Autónoma de Buenos Aires. Limita, además, al sur con el partido de Quilmes, al oeste con el partido de Lanús y al este con el río de La Plata. El partido está compuesto por siete localidades que agrupan a unos 330.000 habitantes en una superficie que alcanza los $54 \mathrm{~km}^{2}$. Los representantes del poder político local suelen presentar a Avellaneda como uno de los municipios más importantes de la provincia de Buenos Aires por su ubicación geográfica, historia, patrimonio cultural y deportivo, y su desarrollo industrial y comercial.

Luego de la apertura democrática en 1983, representantes de distintas fuerzas políticas ocuparon la Intendencia (Unión Cívica Radical, Alianza y Partido Justicialista). No fue hasta 2011, con la asunción del actual intendente, el ingeniero Jorge Ferraresi, que el deporte adquirió mayor legitimidad debido a la creación de la Secretaría de Deportes y Relaciones con la Comunidad ${ }^{3}$.

El secretario a cargo de esta explicó las ganancias del caso durante una conversación personal: “[...] participación en el gabinete, sentarse con el intendente en las reuniones anuales de gestión, buscar consenso en políticas deportivas... tener recursos humanos y dinero". "Aliento" y "Alentar" son dos programas de dicha secretaría.

El funcionario agregó también en dicha conversación que "Aliento" es un incentivo deportivo cuyo objetivo es "colaborar con el desarrollo de la carrera de deportistas de alto rendimiento de la ciudad, quienes compiten en distintas disciplinas". El programa de incentivo a los deportistas amateurs consiste en la entrega de una ayuda para "financiar parte o la totalidad de la participación en competencias de distinta índole, según la capacidad de cada deportista de forma particular". Los deportistas que reciben este incentivo no se dedican a la práctica de fútbol, que es el deporte profesional

\footnotetext{
2 Por medio de la cursiva se indican los términos nativos (que una vez presentados tendrán el formato normal del texto) y a las comillas para las expresiones de los actores.

3 Dentro de la estructura orgánica de la municipalidad también se encuentran las siguientes secretarías: Privada; Gobierno; Seguridad; Obras y Servicios Públicos; Hacienda y Administración; Legal y Técnica; Salud; Producción, Política Ambiental y Empleo; Política Social y Desarrollo Humano; Cultura; Educación y Promoción de las Artes.
} 
por excelencia en Argentina ${ }^{4}$. Ellos practican natación, taekwondo, patín artístico, pádel, judo, boxeo, lanzamiento de martillo, triatlón, equitación, pentatlón moderno, tenis, bocha (paralímpica) y atletismo. Cuando un joven futbolista, que tal vez comienza su práctica desde pequeño en un club de barrio o sociedad de fomento, expone su talento, es convocado por una institución que financia los costos de su carrera (viáticos, indumentaria, pasajes). Un futbolista que logra insertarse finalmente en el mercado laboral, profesional deportivo, pauta con el club que representa un sueldo por su actuación. La carrera de los atletas de otras disciplinas es distinta: transitan en el amateurismo, con un sostén básico de sus clubes (que refiere principalmente a la logística de las competencias y al espacio físico) y sin el apoyo económico del sector privado. Cuando los atletas se dedican a deportes amateurs resulta una prioridad conseguir el aporte empresarial. Como me explicó el nadador paralímpico, Ariel Quassi:

[...] el tema es cultural ya que en otros países se acostumbra a que las empresas patrocinen deportistas, lo cual tiene un beneficio impositivo. Lamentablemente en nuestro país, por distintas cuestiones, las empresas no enfocan sus intenciones en mostrarse de esta manera, sumados a la política impositiva y su reiterada evasión por parte de quienes deben aportar, resulta muy negativo para el deporte en sí.

En una entrevista realizada por un medio partidario del Club Atlético Independiente en 2011 resaltó:

[...] tengo la suerte de que me va bien, y puedo dedicarme a esto [...]. Entreno dos veces por día, de lunes a sábados, me levanto a la mañana, vengo a entrenar al club (Independiente), después vuelvo a mi casa, descanso, y vuelvo a entrenar. [...] Hay muchísimas cosas para hacer, me gustaría poder trabajar para que los deportistas tengan más comodidades de las que pude haber tenido yo al momento de entrenar y competir. Hoy lo que falta es apoyo económico. Hace poquito pudimos conseguir un plan de la municipalidad de Avellaneda que nos va a ayudar para salir a competir, pero la problemática del deportista argentino es el día a día. Esto es un trabajo para mí, si no tengo becas, sponsors y entradas mensuales, el entrenamiento diario se hace muy complicado. Nosotros tenemos muchos gastos de movilidad, suplementos, indumentaria.

4 El profesionalismo surgió en 1931.
Y se hace imposible sin apoyo económico. Para ir a competir y obtener buenos resultados se necesita entrenar, y para entrenar se necesita tiempo. Entonces, esto es un trabajo. Los deportistas trabajamos de deportistas. Entonces, necesitamos apoyo mensual y es lo más complicado para conseguir. Hace 14 años que me dedico full time a esto. Esto es una forma de vida [...]. (Entrevista realizada por un socio del Club Atlético Independiente y publicada en el canal del autor en YouTube) ${ }^{5}$.

Del fragmento de la entrevista se desprende una situación particular, que se multiplica entre los atletas argentinos: deportes que son clasificados por sus federaciones como amateurs reúnen en su seno a deportistas que forjan sus carreras con una dedicación total o parcial para llegar al alto rendimiento. ¿Cómo financian estos deportistas sus entrenamientos y competencias? Ariel, como nadador de la Selección Argentina, percibe una beca de la Secretaría de Deportes de la Nación y del ENARD ${ }^{6}$. Además, goza del apoyo de un sponsor, el sindicato de la Asociación Personal Técnico Aeronáutico (APTA). Asimismo, en sus tiempos libres da clases en la Universidad Argentina de la Empresa, en la carrera de publicidad, dos veces por semana, y colabora en la Secretaría de Deportes y Relaciones con la Comunidad de Avellaneda como coordinador del programa "Alentar". Por eso, como me explicó en una conversación personal:

[...] debo ser de los deportistas que menos recurren al programa Aliento y se debe a que por estar entre los 8 mejores del mundo y ser parte de la Selección Argentina tengo varios de mis torneos cubiertos, por eso suelo recurrir sólo una vez por año para solicitar la colaboración para participar de algún viaje, pero intento hacerlo solo cuando es estrictamente necesario.

5 Entrevista publicada en https://www.youtube.com/ watch?v=JHWQ6rEliN8

Ariel Quassi participó de los últimos tres Juegos Paralímpicos. Fue campeón argentino durante 11 años -1997 a 2009 de forma consecutiva- y posee el récord mundial de los 50 metros, estilo pecho en pileta corta. Además, fue diploma olímpico en Sídney, Grecia y Beijing.

6 El Ente Nacional de Alto Rendimiento Deportivo comenzó a funcionar en 2010 (reglamentación de 2009) para potenciar el crecimiento y la consolidación del deporte nacional. La página oficial explica que sus socios fundadores y coadministradores son el Comité Olímpico Argentino (COA) y la Secretaría de Deportes del Ministerio de Desarrollo Social de la Nación. Los recursos para financiar los proyectos surgen del cargo del $1 \%$ aplicado sobre el abono que las empresas de telefonía celular facturan a sus clientes por los servicios de telecomunicaciones brindados (Ente Nacional de Alto Rendimiento Deportivo, 2014). 
La situación de Ariel también la explica el secretario del área del municipio: "[...] cuando tiene que viajar y el Club Atlético Independiente no lo puede ayudar, entonces el municipio lo ayuda con el costo de su traslado". Precisamente, debido a los problemas de financiamiento que genera el amateurismo, los funcionarios del Gobierno municipal sostienen que intentan gestionar el acceso a recursos que faciliten su participación en competencias nacionales e internacionales. En este sentido, la implementación del programa incluye la articulación con empresas locales para que actúen como sponsors de los atletas.

Los deportistas del programa "Aliento", como tantos otros en Argentina, consideran que sus carreras están atravesadas por el sacrificio. Esta categoría es comúnmente usada por los atletas para expresar una serie de adversidades que deben sortear día tras día en su devenir competitivo; adversidades de toda índole que van desde juntar dinero para viajar al lugar de la competencia (en el país o en el exterior), encontrar un espacio apropiado para entrenar con el equipamiento necesario, trabajar con entrenadores indicados para sus objetivos, gozar de atención médica, hasta cuestiones relativas al uso del tiempo libre que implica ausentarse de eventos sociales (cumpleaños, reuniones con amigos, asados familiares). La mayor parte de los 60 integrantes que componen el programa "Aliento" entrenan algunas horas antes o después de su jornada laboral. Su situación no es la que experimenta Ariel Quassi si consideramos el apoyo multisectorial que recibe hoy el nadador. No obstante, estos deportistas -Ariel incluidopiensan sus trayectorias en términos de sacrificio. Esta categoría ha sido analizada por Julia Hang (2011) para los nadadores máster ${ }^{7}$

Hacer el sacrificio significa para los máster trabajar para poder reunir la cantidad de dinero suficiente para viajar a los torneos, pero también implica que, a pesar de las obligaciones que la vida adulta $y$ familiar trae consigo, poder organizarse para entrenar y competir, postergar algunos disfrutes en función de afrontar los gastos de los viajes a los torneos. A su vez hay un sentido de sacrificio que se

7 La autora cita un documento de la Federación Internacional de Natación para explicar que "el programa de Máster será promover la salud, la amistad, la comprensión y la competencia a través de natación, saltos, natación sincronizada, waterpolo y natación en aguas abiertas, entre los competidores con un límite mínimo de edad de 25 años (con la salvedad de la inclusión de la categoría "Pre-Master" de 20 a 25 años)". asocia a lo corporal, al agotamiento, al sufrimiento, a la imposición de ir a entrenar más allá de estar cansado por las obligaciones diarias, de madrugar con el solo objetivo de ir a entrenar o bien de tirarse al agua a pesar del frío [...]. Para los máster, entonces, la idea de sufrir y sacrificarse en el entrenamiento, funcionará por un lado como una apuesta a futuro, como una inversión que tendrá sus frutos en la competencia, pero además, como marca de superioridad moral, que hermanará a los nadadores que han compartido y transitado una experiencia de sacrificio en el agua (pp. 121-122).

Asimismo, vale recordar el texto de Wacquant (2006) sobre los boxeadores amateurs, habitantes de un gueto negro de Chicago, donde el sacrificio aparece unido a tres condiciones que los atletas deben cumplir para participar de forma óptima en sus combates: cuidar la alimentación, abstenerse de las relaciones sexuales antes de las peleas y limitar los encuentros sociales.

Entre los deportistas que figuran en el programa "Aliento" se destaca a la boxeadora Yésica Bopp, que reúne características particulares. La Tutti, como le dicen, forjó su carrera profesional desde 2008 después de pasar por el amateurismo y, como toda atleta profesional de este deporte, recibe una remuneración por pelea, que es previamente acordada entre su representante y el promotor que organiza el combate. La boxeadora desde sus inicios como profesional recibe el apoyo económico para dedicarse exclusivamente al boxeo de la Asociación de Trabajadores de la Industria Lechera de la República Argentina (Atilra) ${ }^{8}$. Asimismo, en la actualidad, tiene sponsors del sector privado que le aseguran una entrada de dinero a cambio de la publicidad que ella hace de la marca en sus presentaciones públicas. Yésica es campeona mundial de la categoría minimosca de la Asociación Mundial de Boxeo y de la Organización Mundial de Boxeo, y de la categoría mosca de la OMB. Por supuesto, debido a su posición, ella no precisa la ayuda del programa "Aliento".

La boxeadora es una deportista consagrada y es la cara visible de distintas actividades y proyectos que enuncia la Secretaría de Deportes y Relaciones con la Comunidad de Avellaneda. Ella suele participar como invitada estelar de variados eventos que organiza la municipalidad (una clínica de boxeo, la inauguración de las obras de un club, cenas y homenajes). En la actualidad

\footnotetext{
8 Atilra apoya a otros boxeadores de envergadura. Entre otros sindicatos de trabajadores que promueven el deporte, se encuentra también el Sindicato de Choferes de Camiones.
} 
integra, junto al nadador Ariel Quassi, el Consejo de Alto Rendimiento Deportivo que, como explicó este último,

[...] tiene como objetivo, a partir del reclutamiento de especialistas en áreas específicas del deporte de la ciudad ${ }^{9}$ poder capacitar y potenciar a los entrenadores y deportistas de la ciudad, supervisar instalaciones existentes y a construir, generar un vínculo por el cual desde el deporte social se llegue a una base importante para luego trabajar el alto rendimiento deportivo.

En relación con el programa "Aliento", Ariel contó que "en un principio (los deportistas) eran sólo de alto rendimiento y luego por una cuestión de necesidades se amplió un poco más, tratando de organizar a todos los deportistas por niveles para que sea más efectiva la ayuda en sí", pues hay deportistas de distintas edades y categorías: adultos, juveniles y cadetes. El programa, que no recibe financiamiento del presupuesto del Gobierno nacional, sino que funciona de acuerdo con lo recaudado en una cena anual que se realiza a fin de año entre los empresarios del municipio, tiene como criterios de elección: la calidad y el hecho de ser de Avellaneda.

Si el programa "Aliento" está pensado como una ayuda a deportistas de élite (o con condiciones para lograr ese nivel), el programa "Alentar" está destinado a, según la página oficial del municipio al deporte recreativo: "[...] fortalecer a las instituciones deportivas, devolviéndoles un rol fundamental en nuestra sociedad [...] cada semana se inauguran nuevas e importantes obras en los clubes de barrio de la ciudad" (Revista MdA, 2011, p. 10). El municipio cuenta con 160 clubes de barrio (124 activos), que son instituciones sociales y deportivas de menor envergadura que los clubes con fútbol profesional ${ }^{10}$. La ayuda del programa "Alentar" se cristaliza en materiales para la práctica deportiva (pelotas, redes, palos de hockey) y también en el acondicionamiento de las instituciones (pintura, refacción, construcción de techos, baños, gimnasios, pisos, carpintería, instalación eléctrica). El propósito es revitalizar los clubes de barrio, que durante la década de 1990

9 Yésica Bopp en boxeo, Ariel Quassi en deporte paralímpico, Maxi Schell en pentatlón moderno, Daniel Gómez en atletismo y Román López en el área dirigencial deportiva.

10 No obstante, hay que considerar que el Club Atlético Independiente, Racing Club y Arsenal Fútbol Club en su calidad de asociaciones civiles brindan una variada oferta de deportes amateurs y actividades sociales. sufrieron la envestida del modelo neoliberal caracterizado por el achique de las funciones del Estado. El intendente comentó en una entrevista publicada en un diario del municipio: "Queremos recuperar todos los clubes de barrio para alegría de nuestros chicos y que en estos espacios se trabaje de manera solidaria con los vecinos" (Diario La Ciudad, 20 de octubre de 2010).

En el mismo sentido, el secretario de Deportes y Relaciones con la Comunidad mencionó: “[...] los clubes para nosotros son la célula primaria del deporte social". En conversación, el funcionario explicó que en los clubes de barrio y en los cinco polideportivos del municipio funcionan las escuelas de atletismo y las 90 escuelas que ofrecen deportes como patín, canotaje, skate, boxeo, judo y demás disciplinas. Al mismo tiempo, el secretario manifestó que al observar que en las escuelas municipales solo había varones haciendo deportes, con excepción de las escuelas de fútbol femenino, decidieron organizar las ligas municipales de deportes como handball, hockey y vóley para incorporar a las mujeres. Por otra parte, para establecer una distinción respecto de la política deportiva de otro municipio del Conurbano Sur, el Secretario comentó: “[...] conozco cómo es en Quilmes... ellos financian la liga de fútbol, pagan árbitros, el traslado [...]”. Es decir, los proyectos del municipio vecino estarían destinados principalmente al desempeño del fútbol infantil, que es el deporte que más convocatoria tiene en nuestro país.

Para los funcionarios de Avellaneda, las ligas recreativas y las escuelas están destinadas a la inclusión amplia de los niños y jóvenes del municipio. El deporte es entendido como un derecho: todos aquellos que quieran participar de las actividades planificadas por el programa "Alentar" pueden hacerlo. Para esto, los responsables crean un escenario de oportunidades a través del acceso gratuito a la práctica deportiva y la oferta descentralizada de disciplinas distintas, distribuidas en todo el territorio local. En este repertorio, las categorías deporte recreativo y deporte social actúan como sinónimos, pues ambas son usadas en términos de inclusión, integración y derecho, donde no se privilegia la calidad sino el jugar por jugar. No obstante, las categorías conllevan una contradicción de la que dio cuenta el mismo secretario que risueñamente indicó: “[...] bueno... recreativo, pero cuando están jugando quieren ganar" (y a veces en pos de ganar, los 
equipos se arman entre aquellos que tienen mejores condiciones).

Un aspecto complementario que hace funcionar el programa "Alentar" es el de los recursos humanos. En este sentido, el programa ofrece pasantías educativas del Instituto de Educación Física ubicado en el municipio (Instituto Superior de Formación Docente N. ${ }^{\circ} 101$, que depende de la Provincia de Buenos Aires). Cada año, 60 jóvenes egresados reciben una beca de $\$ 750$ para dar clases en las escuelas municipales dos veces por semana por dos horas. Es un convenio para que los alumnos que egresan del profesorado inicien su trayectoria laboral.

Para el secretario de Deportes de Avellaneda, la enseñanza y la práctica regular de distintos deportes en el municipio podrían producir otras consecuencias positivas. "El deporte social necesita algo más. Los políticos compran materiales para la iniciación deportiva (por ejemplo, un palo de hockey que cuesta $\$ 100$ ), pero no lo compramos siempre, lo compramos una vez". Si después las chicas quieren seguir jugando, se espera que las familias hagan el esfuerzo de comprar uno nuevo si lo necesitan. Las chicas que terminan la escuela en alguna institución y quieren seguir jugando "no pueden ir al Regatas, que es el más elitista de Avellaneda, tampoco a Arsenal (Fútbol Club) que tiene una cuota alta". Entonces la idea del secretario es que aquellas que quieran seguir jugando puedan hacerlo en el Polideportivo Sarmiento, ubicado en la localidad de Villa Domínico. Lo mismo piensa para un deporte como el vóley. El secretario imagina la posibilidad de formar

[...] una selección de vóley de Avellaneda que tal vez no juegue en la Liga de Honor, que juegue siempre en la $\mathrm{D}$, pero que las chicas sigan jugando, y una vez que entran en el circuito, se van vinculando y pasando a otros clubes. Se arma el intercambio de jugadoras entre instituciones. A Bolívar ${ }^{11} \ldots$ ipor qué se lo conoce?..., por su vóley.

Así, el deporte funcionaría no solo como un espacio de integración e inclusión de los jóvenes sino también como un medio para promocionar la gestión

11 El Club Ciudad de Bolívar es de San Carlos de Bolívar, de la provincia de Buenos Aires, que se formó en 2002 para competir en la liga más importante de vóley. Los logros sostenidos del equipo llevaron a que la ciudad fuera conocida masivamente. Otro ejemplo es el equipo Loma Vóley, que es una formación que representa al Partido de Lomas de Zamora, ubicado en el Conurbano Sur. gubernamental y la imagen de los responsables de los proyectos políticos. Los deportistas podrían llevar a distintos lugares la marca registrada del partido.

\section{ARTICULACIONES CON LA POLÍTICA NACIONAL}

En 2011, Ferraresi ganó con holgura las elecciones municipales en representación del Partido Justicialista, que era (y sigue siendo) la fuerza política central del Frente Para la Victoria (FPV), liderado en un primer momento por Néstor Kirchner, y en la actualidad por la Presidenta de la Nación, Cristina Fernández de Kirchner.

En rigor, después de casi 15 años al frente de la Secretaría de Obras y Servicios Públicos (1991-1999 y 2003-2009) de Avellaneda, el ingeniero Ferraresi desempeñó el cargo de intendente interino entre 2009 y 2011 en reemplazo del renunciante intendente que dejó su función para sumarse al Ministerio de Desarrollo Social de la Provincia de Buenos Aires. Ferraresi es un intendente kirchnerista, como se conoce a los políticos que adhieren al Gobierno nacional. Este se distingue por una serie de medidas progresistas que fueron elaboradas para solucionar problemas de distinta índole, que resulta complicado detallar aquí12. No obstante, para contextualizar el argumento de este trabajo, se nombran solo algunas decisiones gubernamentales. Durante los años transcurridos desde la asunción del expresidente de la Nación Néstor Kirchner en 2003, se impulsaron las siguientes medidas: extensión de los derechos jubilatorios a alrededor de dos millones de personas cuyos aportes previos habían sido irregulares o nulos; otorgamiento de la Asignación Universal por Hijo para los niños y jóvenes de los sectores más vulnerables; televisación del fútbol profesional por los canales abiertos; promulgación de la ley de matrimonio igualitario entre personas del mismo sexo y la ley de identidad de género, que permite que personas trans(travestis, transexuales y transgéneros) sean inscriptas en sus documentos personales con el nombre y el sexo de elección; creación de Universidades Nacionales en distintos puntos del país (como la Universidad Nacional de Avellaneda). Estas y otras decisiones fueron

12 Puede consultarse Romero (2012) para observar el estilo de gobierno desde una perspectiva crítica. 
pensadas como propuestas generadoras de igualdad, inclusión e integración.

La Secretaría de Deportes y Relaciones con la Comunidad de Avellaneda funciona en un edificio reciclado que lleva el nombre 27 de Abril-Néstor Kirchner. Allí, cuando le pregunté al Secretario del área en qué casos se habían inspirado para formular los programas "Aliento" y “Alentar", explicó que era

[...] inspiración del intendente, que la propuesta había nacido desde la asunción de Jorge y que el trabajo municipal se basaba mucho en las instituciones, pero que, lógicamente todos nos inspiramos en un Gobierno central, con el que empezamos a entender las desigualdades.

Así, las políticas públicas deportivas municipales se insertan en la línea interpretativa de las políticas de orden nacional en distintos ámbitos (salud, educación, desarrollo social, trabajo, etc.) que se basan en "la ampliación de derechos y oportunidades".

Las articulaciones con la política nacional son múltiples $^{13}$. En el caso del deporte, observamos la coincidencia de una serie de ideales que se cristalizan en políticas públicas definidas, y en convenios concretos entre el Gobierno municipal y el Gobierno nacional. En este sentido, vale aclarar que la Secretaría de Deportes de la Nación funciona bajo la órbita del Ministerio de Desarrollo Social del Gobierno central. Esto implica pensar, desde el punto de vista de los funcionarios, que el deporte es un espectáculo o un entretenimiento, así como también una herramienta del proyecto de inclusión y ampliación de derechos.

En términos de los vínculos entre el Gobierno local y el Gobierno nacional existen programas como, entre otros, el que vincula a las cinco disciplinas de contacto (en Avellaneda) en convenio con el ENARD. En junio de 2013, se inauguró el primer Centro de Deportes de Combate del país en el Gimnasio José María Gatica en la localidad de Villa Domínico. Allí, jóvenes de entre 13 y 16 años practican lucha, karate, taekwondo, boxeo y judo, en un espacio que cuenta con dos tatamis, un ring de box, bolsas de boxeo, pesas y aparatos de musculación. Representantes de distintos espacios de la política (local y nacional) y espacios deportivos (de las

13 Para tratar en profundidad las articulaciones entre la política municipal, provincial y nacional en torno a proyectos deportivos recreativos en la ciudad de La Plata, ver Levoratti (2013). federaciones de los deportes involucrados) participaron de la inauguración del Centro. Entre los objetivos del emprendimiento se mencionaron: "[...] acompañar la evolución de los deportistas y ampliar la base para detectar jóvenes valores procurando la igualdad de posibilidades para todos". El intendente del municipio expresó:

Para algunos esta obra es un gasto pero para nosotros es una gran inversión. Trabajamos permanentemente con los clubes y deportistas de nuestro municipio y en este lugar que se inaugura hoy vamos a preparar deportistas para los Juegos Olímpicos. Sabemos que hay muchos jóvenes que tienen la capacidad de ganar las olimpíadas pero quizás no tienen el respaldo, entonces aquí estamos nosotros como Estado, para darles las herramientas necesarias para cumplir sus sueños [...]. Este Centro es el primero del país que se lanza para que los chicos sean representantes olímpicos, por eso le agradecemos a la presidenta Cristina Fernández; a la ministra de Desarrollo Social, Alicia Kirchner; a la Secretaría de Deporte de la Nación y al ENARD. Trabajamos en la inclusión y el desarrollo de los chicos. Esta política deportiva la creamos para que perdure y por eso acompañamos a la presidenta en este liderazgo de la Argentina (La Noticia del Sur, 4 de junio de 2013).

Por último, el Gobierno nacional posibilita la canalización de dinero para invertir en grandes obras. De allí salieron los fondos para recuperar el polideportivo Sarmiento en el Parque Domínico donde se estableció la agrupación de atletismo Osvaldo Suárez, y para la construcción del Club Nueva Esperanza 2000, en uno de los barrios más pobres del partido, Villa Tranquila. Frente a la obra en construcción figura el siguiente cartel: Aquí la nación también crece.

\section{CONCLUSIÓN}

Los significados que rodean a los programas "Aliento" y "Alentar", que impulsa el intendente que adhiere al modelo kirchnerista, deben entenderse en el entramado de significados que surgen de las políticas públicas del Gobierno nacional. Que el deporte se haya convertido para el gobierno municipal en un espacio para la producción de políticas públicas -con tales sentidosno es una casualidad. Avellaneda es un partido donde conviven reconocidos clubes de fútbol, de distintas divisiones, que ofrecen en su calidad de instituciones 
sociales y deportivas la posibilidad de practicar disciplinas amateurs y realizar actividades recreativas. Avellaneda, además, se destaca por la vigencia de 124 clubes de barrio, ubicados en distintos puntos del territorio comunal. Así, ambos aspectos se conjugan en esta coyuntura: el deporte como política de inclusión, integración e igualdad para los habitantes del municipio.

El programa "Aliento" está destinado a los atletas de alto rendimiento que quieren posicionarse en los mejores puestos de las competencias nacionales e internacionales. Los funcionarios presentan los beneficios del programa y, en dicho proceso, exponen las dificultades que encuentran los atletas cuando quieren dedicarse a la práctica full time de un deporte amateur. Ellos -y los deportistas también- dan cuenta de la falta de recursos económicos para el desarrollo de las carreras deportivas. Muestran así que el deporte de alto rendimiento se realiza bajo condiciones de carencia. Las políticas públicas del Gobierno municipal intentan superar alguna de las dificultades que viven los atletas, financian una participación por año en una competencia a cada uno de ellos. Lo hacen recurriendo a la donación de dinero de las empresas de Avellaneda. Por lo general, la dificultad que tienen los deportistas amateurs para conseguir auspiciantes del sector privado se funda en la escasa cobertura que los medios de comunicación hacen de sus actuaciones.

Los deportistas de alto rendimiento han señalado que la práctica del deporte es un trabajo. Si bien la categoría no fue desarrollada profundamente en este artículo, la misma sugiere algunos sentidos. Por un lado, el deseo del deportista de dedicarse al deporte de forma exclusiva. Esto es, vivir para y del deporte. Una meta que todos no logran. Por otro lado, el sacrificio que se expresa en términos del esfuerzo económico y físico que deben afrontar día tras día, en circunstancias desfavorables, antes o después de su trabajo efectivo.

El caso del nadador paralímpico Ariel Quassi es especial porque recibe el apoyo económico del ENARD y de la Secretaría de Deportes de la Nación, así como también de un sponsor. Ariel, a la inversa de la mayoría, trabaja -como profesor de publicidad en la Universidad de la Empresa- en su tiempo libre. Es profesor cuando no entrena. Su verdadero trabajo es la natación. Para Yésica, que es una boxeadora exitosa, la situación también es favorable y distante de la situación de los atletas del programa que no gozan de becas ni sponsors. No obstante, para los deportistas que se dedican al deporte de élite, la condición sigue siendo la del sacrificio por la serie de adversidades que afrontan o debieron afrontar durante la trayectoria de sus carreras.

El presente trabajo muestra una situación que atraviesa el deporte de élite en Argentina: ¿Cómo financian los atletas, que se dedican a deportes amateurs, sus entrenamientos y competencias? El Gobierno nacional ayuda a un sector de los deportistas de alto rendimiento a través de becas y subsidios. También pauta convenios con los municipios en nombre de los valores e ideales presentados más arriba, como la apertura de centros de entrenamiento y capacitación. Estos lugares se piensan en un doble sentido: como espacios para la búsqueda de talentos y como espacios que amplían las oportunidades para hacer deporte.

El deporte social o recreativo se conecta con el deporte de élite pues, como dijo Ariel Quassi, un objetivo del Consejo Deportivo de Alto Rendimiento de Avellaneda es "generar un vínculo por el cual desde el deporte social se llegue a una base importante para luego trabajar el alto rendimiento deportivo". Como se desprende del objetivo del Concejo y de la puesta en práctica del deporte recreativo en los clubes de barrio y en las escuelas municipales, el programa "Alentar" expone sus contradicciones. Si bien el programa valora positivamente la inclusión e integración de niños y jóvenes de todas las condiciones y orígenes a partir del objetivo jugar por jugar, estos principios pueden encontrar sus límites en el deseo de ganar y en la elección de los mejores para competir. 


\section{REFERENCIAS BIBLIOGRÁFICAS}

Hang, J. (2011).Sacrificio y sociabilidad en el entrenamiento de nadadores máster. Educación Física y Ciencia, 13, 111-125.

Diario La Ciudad, Inauguraron obras en el Club Albión, en el marco del Programa Municipal Alentar. Recuperado de http://laciudadavellaneda.com.ar/inauguraronobras-en-el-club-albion-en-el-marco-del- programamunicipal-alentar/

Ente Nacional de Alto Rendimiento Deportivo, 2014.

La Noticias del Sur, Avellaneda: inauguran el primer Centro de Especialización en Deportes de Combate en Argentina, 4 de junio de 2013. Recuperado en http://www.lanoticiasur.com.ar/despachos.asp?cod_ des $=16100 \&$ ID_Seccion $=178$
Levoratti, A. (2013). Disputas por el sentido del deporte en programas de política socio-educativa. Un análisis sobre sus representaciones y prácticas en funcionarios estatales y profesores de educación física (Provincia de Buenos Aires 2004-2011). Tesis inédita de maestría. Buenos Aires: IDES-IDAES/Universidad Nacional de San Martín, Argentina.

Revista Municipalidad de Avellaneda (junio de 2011), año VII, N. -18.

Romero, L.A. (2012). Breve historia contemporánea de la Argentina. Buenos Aires: Fondo de Cultura Económica.

Wacquant, L. (2006). Entre las cuerdas. Cuadernos de un aprendiz de boxeador. Buenos Aires: Siglo XXI. 